\title{
Eleven New 2-Pyrones from a Fungi Imperfecti, Trichurus terrophilus, Found in a Screening Study Guided by Immunomodulatory Activity
}

\author{
Haruhiro Fujimoto, $\left.{ }^{*}, 1\right)$ Yoshie Okamoto, Eriko Sone, Satomi Maeda, Kohki AkiYama, and \\ Masami ISHIBASHI \\ Graduate School of Pharmaceutical Sciences, Chiba University; 1-33 Yayoi-cho, Inage-ku, Chiba 263-8522, Japan. \\ Received February 7, 2005; accepted April 14, 2005
}

In a screening study on immunomodulatory fungal constituents, eleven new 2-pyrones tentatively named TT-1 (1), and TT-2 - 11 (2-11) have been isolated from a Fungi Imperfecti, Trichurus terrophilus, and designated rasfonin (1), and trichurusin B-K (2-11), respectively. Compounds $1-4$ exhibited considerably high immunosuppressive activities, and compounds 8-11 have shown moderate ones. The structure-activity relationships of these constituents have also been discussed.

Key words immunosuppressive fungal constituent; Trichurus terrophilus; Fungi Imperfecti; 2-pyrone; trichurusin; structureactivity relationship

In our screening program on immunomodulatory constituents from fungi, many immunosuppressive metabolites have already been isolated from various Ascomycetes belonging to Gelasinospora, Diplogelasinospora, Microascus, Emericella, Eupenicillium, Chaetomium, and Zopfiella. ${ }^{2)}$ The EtOAc extract of a Fungi Imperfecti, Trichurus terrophilus SwIFT \& POVAH showed an appreciable suppressive effect on the proliferation (blastogenesis) of mouse splenic lymphocytes stimulated with mitogens, concanavalin A (Con A), and lipopolysaccharide (LPS). Solvent partitions followed by repeated chromatographic fractionations of the extract, guided by immunosuppressive activity, afforded eleven new compounds tentatively named TT-1-11 (1-11). This paper deals with the structures and immunosuppressive activities of these constituents.

The EtOAc extract of T. terrophilus IFM4606 ${ }^{3)}$ cultivated on sterilized moistened-rice medium suppressed the Con Ainduced proliferation of mouse splenic lymphocytes by $99.6 \%$ at $50 \mu \mathrm{g} / \mathrm{ml}$. The EtOAc extract was partitioned between $n$-hexane and water into an $n$-hexane layer and an aqueous suspension. The aqueous suspension was further partitioned between EtOAc and water into an EtOAc layer and an aqueous layer [yields (\%) of the $n$-hexane, EtOAc, and aqueous layers after evaporation of the solvents from the EtOAc extract: $73.2,17.4$, and 2.3, respectively]. The $\mathrm{IC}_{50}$ values of the $n$-hexane, EtOAc, and aqueous layers against the Con A-induced proliferation were 9.7, 2.5, and $>50 \mu \mathrm{g} / \mathrm{ml}$, respectively. Repeated chromatographic fractionations monitored by the immunosuppressive activity of the EtOAc layer afforded four components tentatively named TT$1-4(\mathbf{1}-\mathbf{4})$ [yields $(\%)$ of $\mathbf{1}, \mathbf{2}, \mathbf{3}$, and 4 from the EtOAc extract: $0.26,0.0078,0.011$, and 0.0089 , respectively]. It was found out that the other strain of this fungus, T. terrophilus IMI46251 ${ }^{3)}$ also produced $\mathbf{1}$ and its homologues in good yields. Repeated chromatographic fractionations of the EtOAc layer, which was obtained from the partition of the EtOAc extract of T. terrophilus IMI46251 in the same way as described for the partition of the EtOAc extract of $T$. terrophilus IFM4606, gave seven new constituents tentatively named TT-5-11 (5-11) in addition to $\mathbf{1}-\mathbf{4}$ [yields $(\%)$ of $1,2,3,4,5$, a mixture of 6 and $7(6 / 7), 8,9,10$, and 11 from the EtOAc extract: 1.7, 0.00088, 0.0091, 0.0044, 0.0025,
$0.00094,0.0019,0.0036,0.0011$, and 0.00089 , respectively].

TT-1 (1), obtained as an optically active white amorphous powder $\left([\alpha]_{\mathrm{D}}^{24}-223.6^{\circ}\right)$, circular dichroism (CD) in $\mathrm{MeOH}$ (0.11 mM), $\Delta \varepsilon$ (nm): -98 (266), -51 (239), -255 (212), gave $\mathrm{C}_{25} \mathrm{H}_{38} \mathrm{O}_{6}$ as the molecular formula. The ${ }^{1} \mathrm{H}$ - and ${ }^{13} \mathrm{C}$ NMR spectral data of 1 (see Table 1) including the two dimensional ${ }^{1} \mathrm{H}-{ }^{1} \mathrm{H}$ shift correlation (COSY), ${ }^{1} \mathrm{H}$-detected heteronuclear correlation through multiple quantum coherence (HMQC), and ${ }^{1} \mathrm{H}$-detected heteronuclear multiple-bond correlation (HMBC) NMR data suggested a planar structure for TT-1. On acetylation with acetic anhydride in pyridine, $\mathbf{1}$ gave diacetate (12). Comparison of the ${ }^{1} \mathrm{H}$ - and ${ }^{13} \mathrm{C}-\mathrm{NMR}$ spectral data of $\mathbf{1}$ with those of $\mathbf{1 2}$ (Table 1) in reference to the acetylation shift rule, ${ }^{4,5)}$ confirmed the planar structure of TT-1 (1a) (see Fig. 1). TT-2 (2), obtained as an optically active colorless amorphous powder $\left([\alpha]_{\mathrm{D}}^{25}+18.4^{\circ}\right)$, gave $\mathrm{C}_{25} \mathrm{H}_{40} \mathrm{O}_{6}$ as the molecular formula. Comparison of the ${ }^{1} \mathrm{H}$ and ${ }^{13} \mathrm{C}$-NMR spectral data of 2 (Table 1) with those of $\mathbf{1}$ revealed that the unsaturated $\mathrm{C}=\mathrm{C}$ bond at position 3 in $\mathbf{1}$ was saturated in $\mathbf{2}$, indicating that the planar structure of TT-2 was expressed as 2 (Fig. 1). Then, in 2000 we reported the isolation and planar structures of TT-1 (1a) and 2 (2), two new immunosuppressive constituents from T. terrophilus. ${ }^{6}$ Almost at the same time, Hayakawa et al. reported the isolation of rasfonin, a new apoptosis inducer from an Ascomycete, Talaromyces sp. ${ }^{7)}$ The planar structure and the physicochemical properties of rasfonin were the same as those of TT-1. In the absolute structure, the configurations of five chiral centers of TT-1 were determined to be $5 R, 6 R, 7 S, 9 R$, and $6^{\prime} S$ by the synthesis of diastereomers of two fragments of TT-1 (segments A, B) (Fig. 1) in optically active forms, and comparison of their spectral and optical data with those of natural specimens by us in $2003,{ }^{8)}$ and finally by our total synthesis of TT-1 in 2005. ${ }^{9)}$ Thus, the structure of TT-1 was expressed as $\mathbf{1}$ including its absolute stereochemistry, as shown in Fig. 1.

Here, TT-1 (1), and TT-2-11 (2-11) from Trichurus terrophilus were designated as rasfonin, and trichurusin $\mathrm{B}-\mathrm{K}$, respectively. It was decided not to designate TT-1 (1) as trichurusin A because TT-1 was the same to rasfonin.

Trichurusin C (TT-3) (3), obtained as an optically active colorless amorphous powder $\left([\alpha]_{\mathrm{D}}^{25}-166.6^{\circ}\right)$, has $\mathrm{C}_{25} \mathrm{H}_{38} \mathrm{O}_{7}$ 
Table 1. ${ }^{1} \mathrm{H}-$ and ${ }^{13} \mathrm{C}-\mathrm{NMR}$ Data for TT-1 (Rasfonin), TT-1 (Rasfonin) Diacetate, Trichurusin B, Trichurusin J, and Trichurusin $\mathrm{K}$ in $\mathrm{CDCl}_{3}, \delta$ (ppm) from TMS as an Internal Standard

\begin{tabular}{|c|c|c|c|c|c|c|c|c|c|c|}
\hline \multirow{2}{*}{ Position } & \multicolumn{2}{|c|}{ TT-1 (Rasfonin) (1) } & \multicolumn{2}{|c|}{ TT-1 (Rasfonin) diacetate (12) } & \multicolumn{2}{|c|}{ Trichurusin B (2) } & \multicolumn{2}{|c|}{ Trichurusin J (10) } & \multicolumn{2}{|c|}{ Trichurusin K (11) } \\
\hline & $\delta_{\mathrm{H}}$ & $\delta_{\mathrm{C}}$ & $\delta_{\mathrm{H}}$ & $\delta_{\mathrm{C}}$ & $\delta_{\mathrm{H}}$ & $\delta_{\mathrm{C}}$ & $\delta_{\mathrm{H}}$ & $\delta_{\mathrm{C}}$ & $\delta_{\mathrm{H}}$ & $\delta_{\mathrm{C}}$ \\
\hline 2 & & $163.3(\mathrm{~s})$ & & $163.2(\mathrm{~s})$ & & $176.9(\mathrm{~s})$ & & $163.4(\mathrm{~s})$ & & $163.4(\mathrm{~s})$ \\
\hline 3 & $6.21(\mathrm{~d}, 9.8)$ & $124.8(\mathrm{~d})$ & $6.22(\mathrm{~d}, 9.6)$ & $125.0(\mathrm{~d})$ & $2.49(2 \mathrm{H}, \mathrm{m})$ & $28.3(\mathrm{t})$ & $6.21(\mathrm{~d}, 9.6)$ & $125.0(\mathrm{~d})$ & $6.21(\mathrm{~d}, 9.6)$ & $125.0(\mathrm{~d})$ \\
\hline 4 & $7.06(\mathrm{dd}, 9.8,6.1)$ & $140.6(d)$ & $7.05(\mathrm{dd}, 9.6,5.9)$ & $140.5(\mathrm{~d})$ & $1.94(\mathrm{~m}), 2.29(\mathrm{~m})$ & $24.6(t)$ & $7.04(\mathrm{dd}, 9.6,5.9)$ & $140.6(\mathrm{~d})$ & $7.04(\mathrm{dd}, 9.6,5.9)$ & $140.6(d)$ \\
\hline 5 & $5.35(\mathrm{dd}, 6.1,2.5)$ & $61.6(\mathrm{~d})$ & $5.36(\mathrm{dd}, 5.9,2.1)$ & $61.8(\mathrm{~d})$ & $4.75(\mathrm{~m})$ & $79.3(\mathrm{~d})$ & $5.35(\mathrm{dd}, 5.9,2.7)$ & $61.8(\mathrm{~d})$ & $5.35(\mathrm{dd}, 5.9,2.7)$ & $61.8(\mathrm{~d})$ \\
\hline 6 & $4.14(\mathrm{dd}, 8.9,2.5)$ & 83.3 (d) & $4.14(\mathrm{dd}, 8.9,2.1)$ & 83.2 (d) & $4.97(\mathrm{dd}, 5.8,4.1)$ & 76.9 (d) & $4.12(\mathrm{brd}, 8.8)$ & 83.3 (d) & $4.12(\mathrm{brd}, 8.8)$ & $83.4(\mathrm{~d})$ \\
\hline 7 & $2.18(\mathrm{~m})$ & $31.3(\mathrm{~d})$ & $2.17(\mathrm{~m})$ & $31.4(\mathrm{~d})$ & $2.02(\mathrm{~m})$ & 31.9 (d) & $2.16(\mathrm{~m})$ & $31.5(\mathrm{~d})$ & $2.16(\mathrm{~m})$ & $31.5(\mathrm{~d})$ \\
\hline \multirow[t]{2}{*}{8} & $1.04(\mathrm{ddd}, 13.4,9.7,4.5)$ & $39.9(\mathrm{t})$ & $1.04(\mathrm{~m})$ & $39.9(\mathrm{t})$ & $1.00(\mathrm{~m})$ & $40.9(t)$ & $1.03(\mathrm{~m})$ & $40.1(\mathrm{t})$ & $1.03(\mathrm{~m})$ & $40.0(\mathrm{t})$ \\
\hline & $1.20(\mathrm{ddd}, 13.4,9.2,4.0)$ & & $1.23(\mathrm{~m})$ & & $1.36(\mathrm{~m})$ & & $1.23(\mathrm{~m})$ & & $1.23(\mathrm{~m})$ & \\
\hline 9 & $1.69(\mathrm{~m})$ & $27.8(\mathrm{~d})$ & $1.64(\mathrm{~m})$ & 27.9 (d) & $1.71(\mathrm{~m})$ & $28.1(\mathrm{~d})$ & $1.67(\mathrm{~m})$ & $28.0(\mathrm{~d})$ & $1.67(\mathrm{~m})$ & $28.0(\mathrm{~d})$ \\
\hline 10 & $\begin{array}{l}1.44(\mathrm{dd}, 13.2,9.9) \\
2.06(\mathrm{br} \mathrm{d}, 13.2)\end{array}$ & $46.2(\mathrm{t})$ & $\begin{array}{l}1.44(\mathrm{dd}, 13.1,9.8), \\
2.08(\text { br d, 13.1) }\end{array}$ & $46.3(\mathrm{t})$ & $\begin{array}{l}1.63(\mathrm{~m}) \\
2.02(\mathrm{~m})\end{array}$ & $47.2(t)$ & $\begin{array}{l}1.43(\mathrm{~m}) \\
2.06(\mathrm{~m})\end{array}$ & $46.4(t)$ & $\begin{array}{l}1.43(\mathrm{~m}) \\
2.06(\mathrm{~m})\end{array}$ & $46.4(\mathrm{t})$ \\
\hline 11 & & $134.1(\mathrm{~s})$ & & $134.1(\mathrm{~s})$ & & $134.4(\mathrm{~s})$ & & $134.3(\mathrm{~s})$ & & $134.2(\mathrm{~s})$ \\
\hline 12 & $5.12(\mathrm{q}, 6.7)$ & $120.0(\mathrm{~d})$ & $5.12(q, 6.6)$ & $120.0(\mathrm{~d})$ & $5.16(q, 6.3)$ & 120.1 (d) & $5.11(\mathrm{q}, 6.0)$ & 120.2 (d) & $5.11(\mathrm{q}, 6.0)$ & $120.1(d)$ \\
\hline 13 & $1.55(3 \mathrm{H}, \mathrm{d}, 6.7)$ & $13.3(\mathrm{q})$ & $1.54(3 \mathrm{H}, \mathrm{d}, 6.6)$ & $13.3(\mathrm{q})$ & $1.55(3 \mathrm{H}, \mathrm{d}, 6.3)$ & $13.4(\mathrm{q})$ & $1.54(3 \mathrm{H}, \mathrm{d}, 6.3)$ & $13.4(q)$ & $1.54(3 \mathrm{H}, \mathrm{d}, 6.3)$ & $13.4(q)$ \\
\hline 14 & $1.15(3 \mathrm{H}, \mathrm{d}, 6.7)$ & $15.8(\mathrm{q})$ & $1.15(3 \mathrm{H}, \mathrm{d}, 6.4)$ & $15.8(\mathrm{q})$ & $0.95(3 \mathrm{H}, \mathrm{d}, 6.9)$ & $15.7(q)$ & $1.14(3 \mathrm{H}, \mathrm{d}, 6.3)$ & $15.9(q)$ & $1.14(3 \mathrm{H}, \mathrm{d}, 6.3)$ & $15.9(\mathrm{q})$ \\
\hline 15 & $0.78(3 \mathrm{H}, \mathrm{d}, 6.7)$ & $20.5(\mathrm{q})$ & $0.79(3 \mathrm{H}, \mathrm{d}, 6.4)$ & $20.6(q)$ & $0.81(3 \mathrm{H}, \mathrm{d}, 6.6)$ & $20.4(q)$ & $0.77(3 \mathrm{H}, \mathrm{d}, 6.3)$ & $20.7(q)$ & $0.77(3 \mathrm{H}, \mathrm{d}, 6.3)$ & $20.7(q)$ \\
\hline 16 & $1.53(3 \mathrm{H}, \mathrm{s})$ & $15.5(\mathrm{q})$ & $1.53(3 \mathrm{H}, \mathrm{s})$ & $15.5(\mathrm{q})$ & $1.55(3 \mathrm{H}, \mathrm{s})$ & $15.7(q)$ & $1.52(3 \mathrm{H}, \mathrm{s})$ & $15.6(q)$ & $1.52(3 \mathrm{H}, \mathrm{s})$ & $15.6(\mathrm{q})$ \\
\hline $1^{\prime}$ & & $166.1(\mathrm{~s})$ & & $165.9(\mathrm{~s})$ & & $166.8(\mathrm{~s})$ & & $166.1(\mathrm{~s})$ & & $166.1(\mathrm{~s})$ \\
\hline $2^{\prime}$ & $5.80(\mathrm{~d}, 15.6)$ & $114.8(\mathrm{~d})$ & $5.82(\mathrm{~d}, 15.8)$ & $115.6(\mathrm{~d})$ & $5.86(\mathrm{~d}, 15.7)$ & 116.0 (d) & $5.81(\mathrm{~d}, 15.7)$ & 115.4 (d) & $5.81(\mathrm{~d}, 15.7)$ & $115.3(\mathrm{~d})$ \\
\hline $3^{\prime}$ & $7.34(\mathrm{~d}, 15.6)$ & $151.0(\mathrm{~d})$ & $7.33(\mathrm{~d}, 15.8)$ & 150.5 (d) & $7.35(\mathrm{~d}, 15.7)$ & $150.2(\mathrm{~d})$ & $7.34(\mathrm{~d} 15.7)$ & 150.8 (d) & $7.33(\mathrm{~d}, 15.7)$ & $150.9(\mathrm{~d})$ \\
\hline $4^{\prime}$ & & $134.3(\mathrm{~s})$ & & $135.2(\mathrm{~s})$ & & $134.9(\mathrm{~s})$ & & $135.5(\mathrm{~s})$ & & $135.0(\mathrm{~s})$ \\
\hline $5^{\prime}$ & 5.78 (br d, 8.9) & $143.4(\mathrm{~d})$ & $5.69(\mathrm{~d}, 10.1)$ & $141.4(\mathrm{~d})$ & 5.76 (br d, 9.9) & 142.7 (d) & $5.71(\mathrm{~d}, 9.9)$ & 142.7 (d) & $5.71(\mathrm{~d}, 9.9)$ & $142.5(\mathrm{~d})$ \\
\hline $6^{\prime}$ & $2.87(\mathrm{~m})$ & 39.2 (d) & $2.98(\mathrm{~m})$ & $35.3(\mathrm{~d})$ & $2.89(\mathrm{~m})$ & $39.3(\mathrm{~d})$ & $2.84(\mathrm{~m})$ & 38.9 (d) & $3.05(\mathrm{~m})$ & $35.1(\mathrm{~d})$ \\
\hline $7^{\prime}$ & $1.61(\mathrm{~m}), 1.79(\mathrm{~m})$ & $34.8(\mathrm{t})$ & $1.62(\mathrm{~m}), 1.91(\mathrm{~m})$ & $30.5(\mathrm{t})$ & $1.63(\mathrm{~m}), 1.82(\mathrm{~m})$ & $34.9(t)$ & $1.57(\mathrm{~m}), 1.91(\mathrm{~m})$ & $30.4(t)$ & $1.52(\mathrm{~m}), 1.80(\mathrm{~m})$ & $34.4(\mathrm{t})$ \\
\hline $8^{\prime}$ & $3.59(\mathrm{~m}), 3.72(\mathrm{~m})$ & $60.4(t)$ & $3.94(\mathrm{~m}), 4.10(\mathrm{~m})$ & $61.9(\mathrm{t})$ & $3.61(\mathrm{~m}), 3.75(\mathrm{~m})$ & $60.8(t)$ & $3.95(\mathrm{~m}), 4.10(\mathrm{~m})$ & $62.3(\mathrm{t})$ & $3.56(\mathrm{~m}), 3.67(\mathrm{~m})$ & $60.3(\mathrm{t})$ \\
\hline $9^{\prime}$ & $1.83(3 \mathrm{H}, \mathrm{s})$ & $12.5(\mathrm{q})$ & $1.80(3 \mathrm{H}, \mathrm{s})$ & $12.5(\mathrm{q})$ & $1.86(3 \mathrm{H}, \mathrm{s})$ & $12.8(\mathrm{q})$ & $1.81(3 \mathrm{H}, \mathrm{s})$ & $12.7(q)$ & $1.81(3 \mathrm{H}, \mathrm{s})$ & $12.6(q)$ \\
\hline $10^{\prime}$ & $3.59(2 \mathrm{H}, \mathrm{d}, 6.1)$ & $65.6(t)$ & $4.02(2 \mathrm{H}, \mathrm{d}, 6.4)$ & $66.5(\mathrm{t})$ & $3.61(2 \mathrm{H}, \mathrm{m})$ & $66.0(t)$ & $3.56(\mathrm{~m}), 3.62(\mathrm{~m})$ & $65.8(\mathrm{t})$ & $4.03(2 \mathrm{H}, \mathrm{m})$ & $66.9(\mathrm{t})$ \\
\hline \multicolumn{2}{|c|}{$8^{\prime}-\mathrm{OCOCH}_{3}$} & & $2.04(3 \mathrm{H}, \mathrm{s})$ & $20.8(\mathrm{q})$ & & & $2.02(3 \mathrm{H}, \mathrm{s})$ & $21.0(q)$ & & \\
\hline \multicolumn{2}{|c|}{$8^{\prime}-\mathrm{OCOCH}_{3}$} & & & $170.8(\mathrm{~s})$ & & & & $171.0(\mathrm{~s})$ & & \\
\hline \multicolumn{2}{|c|}{$10^{\prime}-\mathrm{OCOCH}_{3}$} & & $2.03(3 \mathrm{H}, \mathrm{s})$ & $20.9(\mathrm{q})$ & & & & & $2.02(3 \mathrm{H}, \mathrm{s})$ & 20.9 (q) \\
\hline \multicolumn{2}{|c|}{$10^{\prime}-\mathrm{OCOCH}_{3}$} & & & $170.8(\mathrm{~s})$ & & & & & & $171.0(\mathrm{~s})$ \\
\hline
\end{tabular}

Multiplicities and coupling constants $(\mathrm{Hz})$ in parentheses.<smiles>CC=C(C)CC(C)CC(C)C1OC(=O)C=CC1OC(=O)C=CC(C)=CC(CO)CCO</smiles>

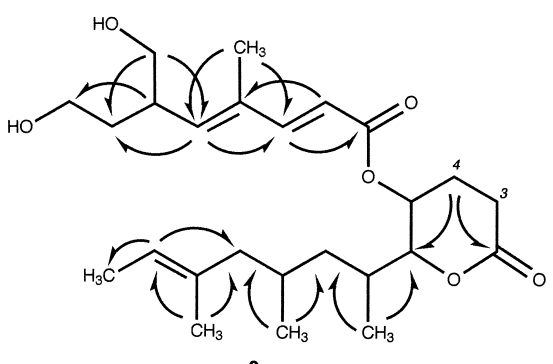

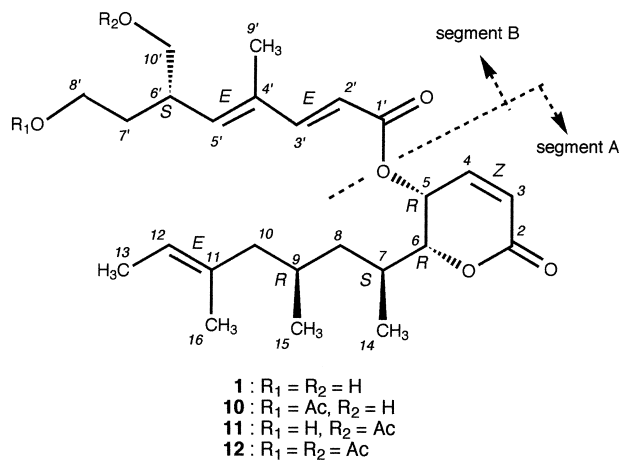

$\overbrace{\mathrm{C}}$ : Selected HMBC $(J=8 \mathrm{~Hz})$

Fig. 1

as the molecular formula. Comparison of the ${ }^{1} \mathrm{H}$ - and ${ }^{13} \mathrm{C}$ NMR spectral data of $\mathbf{3}$ (Table 2) with those of $\mathbf{1}$ showed that instead of the signals of $\mathrm{CH}_{3}-13\left[\delta_{\mathrm{H}} 1.55(3 \mathrm{H}, \mathrm{d})\right.$, $\left.\delta_{\mathrm{C}} 13.3(\mathrm{q})\right]$ and $\mathrm{C}-11\left[\delta_{\mathrm{C}} 134.1(\mathrm{~s})\right]$, those of $\underline{\mathrm{CH}}_{2}=\mathrm{CH}-$
$\left[\delta_{\mathrm{H}} 5.03,5.19(\right.$ each $\left.1 \mathrm{H}, \mathrm{dd}), \delta_{\mathrm{C}} 111.7(\mathrm{t})\right]$ and $>\underline{\mathrm{C}}(-\mathrm{O})$ $\left[\delta_{\mathrm{C}} 73.7(\mathrm{~s})\right]$ newly appeared, suggesting that the group $\mathrm{CH}_{3}-\mathrm{CH}=\mathrm{C}\left(\mathrm{CH}_{3}\right)-$ in segment $\mathrm{A}$ of 1 was replaced with the group $\mathrm{CH}_{2}=\mathrm{CH}-\mathrm{C}\left(\mathrm{CH}_{3}\right)(\mathrm{OH})-$ in that of 3. This was also 


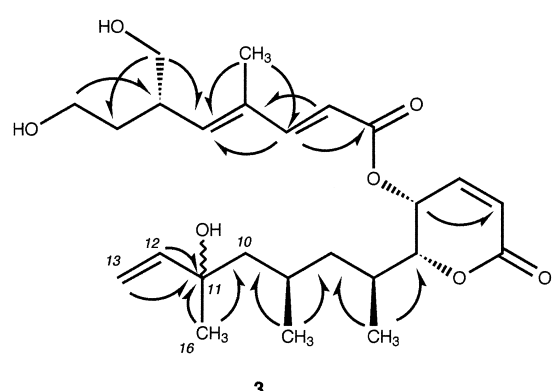

3

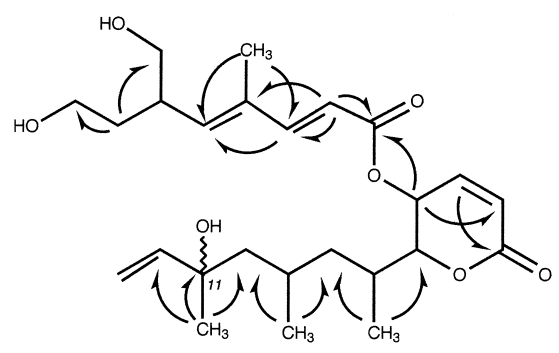

5 (may be the epimer of 3 at position 11)

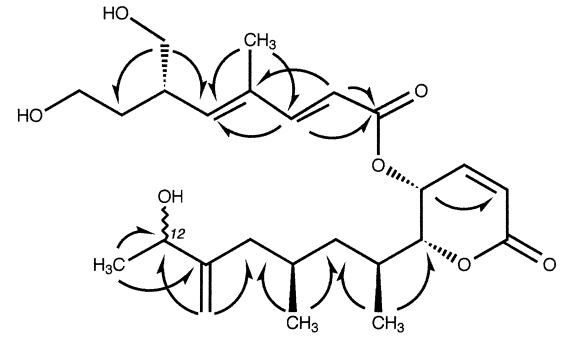

4

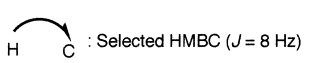

Fig. 2

Table 2. ${ }^{1} \mathrm{H}$ - and ${ }^{13} \mathrm{C}-\mathrm{NMR}$ Data for Trichurusins $\mathrm{C}, \mathrm{D}$, and $\mathrm{E}$ in $\mathrm{CDCl}_{3}, \delta(\mathrm{ppm})$ from TMS as an Internal Standard

\begin{tabular}{|c|c|c|c|c|c|c|}
\hline \multirow{2}{*}{ Position } & \multicolumn{2}{|c|}{ Trichurusin C (3) } & \multicolumn{2}{|c|}{ Trichurusin D (4) } & \multicolumn{2}{|c|}{ Trichurusin E (5) } \\
\hline & $\delta_{\mathrm{H}}$ & $\delta_{\mathrm{C}}$ & $\delta_{\mathrm{H}}$ & $\delta_{\mathrm{C}}$ & $\delta_{\mathrm{H}}$ & $\delta_{\mathrm{C}}$ \\
\hline 2 & & $163.4(\mathrm{~s})$ & & $163.3(\mathrm{~s})$ & & $163.3(\mathrm{~s})$ \\
\hline 3 & $6.21(\mathrm{~d}, 9.6)$ & 125.1 (d) & $6.22(\mathrm{~d}, 9.6)$ & 125.1 (d) & $6.21(\mathrm{~d}, 9.6)$ & $125.1(\mathrm{~d})$ \\
\hline 4 & $7.02(\mathrm{dd}, 9.6,6.0)$ & $140.5(d)$ & $7.02(\mathrm{dd}, 9.6,6.1)$ & $140.4(d)$ & $7.03(\mathrm{dd}, 9.6,5.9)$ & $140.5(d)$ \\
\hline 5 & $5.39(\mathrm{dd}, 6.0,2.5)$ & $61.6(\mathrm{~d})$ & $5.43(\mathrm{dd}, 6.1,2.5)$ & $61.4(\mathrm{~d})$ & $5.42(\mathrm{dd}, 5.9,2.6)$ & $61.5(d)$ \\
\hline 6 & $4.12(\mathrm{dd}, 9.1,2.5)$ & 83.2 (d) & $4.13(\mathrm{dd}, 9.4,2.5)$ & 83.3 (d) & $4.11(\mathrm{dd}, 9.3,2.6)$ & $83.4(d)$ \\
\hline 7 & $2.08(\mathrm{~m})$ & 31.5 (d) & $2.14(\mathrm{~m})$ & 31.7 (d) & $2.11(\mathrm{~m})$ & $31.6(d)$ \\
\hline 8 & $0.94(\mathrm{~m}), 1.25(\mathrm{~m})$ & $41.3(t)$ & $1.05(\mathrm{~m}), 1.25(\mathrm{~m})$ & $40.1(\mathrm{t})$ & $0.94(\mathrm{~m}), 1.23(\mathrm{~m})$ & $41.3(\mathrm{t})$ \\
\hline 9 & $1.62(\mathrm{~m})$ & 26.5 (d) & $1.76(\mathrm{~m})$ & $28.5(\mathrm{~d})$ & $1.63(\mathrm{~m})$ & $26.4(d)$ \\
\hline 10 & $\begin{array}{l}1.19(\mathrm{~m}) \\
1.46(\mathrm{dd}, 14.2,2.6)\end{array}$ & $48.1(t)$ & $\begin{array}{l}1.53(\mathrm{dd}, 14.9,10.2) \\
2.11(\mathrm{dd}, 14.9,3.5)\end{array}$ & $38.8(t)$ & $\begin{array}{l}1.23(\mathrm{~m}) \\
1.52(\mathrm{~m})\end{array}$ & $44.1(\mathrm{t})$ \\
\hline 11 & & 73.7 (s) & & $151.5(\mathrm{~s})$ & & $84.8(\mathrm{~s})$ \\
\hline 12 & $5.83(\mathrm{dd}, 17.3,10.7)$ & $145.2(\mathrm{~d})$ & $4.12(\mathrm{q}, 6.5)$ & $70.6(d)$ & $5.82(\mathrm{dd}, 12.4,10.4)$ & $141.8(\mathrm{~d})$ \\
\hline 13 & $\begin{array}{l}5.03(\mathrm{dd}, 10.7,1.2) \\
5.19(\mathrm{dd}, 17.3,1.2)\end{array}$ & $111.7(\mathrm{t})$ & $1.22(3 \mathrm{H}, \mathrm{d}, 6.5)$ & $22.4(q)$ & $\begin{array}{l}5.17(\text { br d, 10.4), } \\
5.18(\text { br d, 12.4) }\end{array}$ & $115.3(\mathrm{t})$ \\
\hline 14 & $1.12(3 \mathrm{H}, \mathrm{d}, 6.6)$ & $16.2(q)$ & $1.16(3 \mathrm{H}, \mathrm{d}, 6.6)$ & $16.3(q)$ & $1.15(3 \mathrm{H}, \mathrm{d}, 5.5)$ & $16.5(q)$ \\
\hline 15 & $0.96(3 \mathrm{H}, \mathrm{d}, 6.6)$ & $23.3(q)$ & $0.87(3 \mathrm{H}, \mathrm{d}, 6.3)$ & $20.9(q)$ & $0.96(3 \mathrm{H}, \mathrm{d}, 6.6)$ & $23.2(q)$ \\
\hline 16 & $1.20(3 \mathrm{H}, \mathrm{s})$ & $29.2(\mathrm{q})$ & $4.75(\mathrm{~s}), 5.07(\mathrm{~s})$ & $109.5(\mathrm{t})$ & $1.30(3 \mathrm{H}, \mathrm{s})$ & $21.6(q)$ \\
\hline $1^{\prime}$ & & $166.2(\mathrm{~s})$ & & $166.2(\mathrm{~s})$ & & $166.3(\mathrm{~s})$ \\
\hline $2^{\prime}$ & $5.82(\mathrm{~d}, 15.5)$ & $115.1(\mathrm{~d})$ & $5.82(\mathrm{~d}, 15.7)$ & $115.0(\mathrm{~d})$ & $5.83(\mathrm{~d}, 15.6)$ & $115.2(\mathrm{~d})$ \\
\hline $3^{\prime}$ & $7.35(\mathrm{~d}, 15.5)$ & $151.2(\mathrm{~d})$ & $7.36(\mathrm{~d}, 15.7)$ & $151.3(\mathrm{~d})$ & $7.37(\mathrm{~d}, 15.6)$ & $151.2(\mathrm{~d})$ \\
\hline $4^{\prime}$ & & $134.6(\mathrm{~s})$ & & $134.8(\mathrm{~s})$ & & $135.0(\mathrm{~s})$ \\
\hline $5^{\prime}$ & $5.81(\mathrm{~d}, 8.5)$ & $143.8(\mathrm{~d})$ & $5.80(\mathrm{~d}, 11.3)$ & $144.0(d)$ & $5.83(\mathrm{~d}, 6.9)$ & $143.6(d)$ \\
\hline $6^{\prime}$ & $2.87(\mathrm{~m})$ & $39.4(d)$ & $2.87(\mathrm{~m})$ & $39.3(d)$ & $2.90(\mathrm{~m})$ & 39.3 (d) \\
\hline $7^{\prime}$ & $\begin{array}{l}1.58(\mathrm{~m}) \\
1.80(\mathrm{~m})\end{array}$ & $34.8(t)$ & $\begin{array}{l}1.59(\mathrm{dd}, 13.9,8.2) \\
1.79(\mathrm{dd}, 13.9,8.1)\end{array}$ & $34.7(\mathrm{t})$ & $\begin{array}{l}1.60(\mathrm{~m}) \\
1.81(\mathrm{~m})\end{array}$ & $34.6(\mathrm{t})$ \\
\hline $8^{\prime}$ & $3.60(\mathrm{~m}), 3.72(\mathrm{~m})$ & $60.6(\mathrm{t})$ & $3.59(\mathrm{~m}), 3.70(\mathrm{~m})$ & $60.6(\mathrm{t})$ & $3.60(\mathrm{~m}), 3.73(\mathrm{~m})$ & $60.8(\mathrm{t})$ \\
\hline $9^{\prime}$ & $1.83(3 \mathrm{H}, \mathrm{s})$ & $12.7(q)$ & $1.83(3 \mathrm{H}, \mathrm{s})$ & $12.7(q)$ & $1.86(3 \mathrm{H}, \mathrm{s})$ & $12.7(q)$ \\
\hline $10^{\prime}$ & $\begin{array}{l}3.59(\mathrm{dd}, 7.6,6.3) \\
3.60(\mathrm{dd}, 7.6,6.0)\end{array}$ & $65.8(t)$ & $\begin{array}{l}3.57(\mathrm{dd}, 10.7,6.5) \\
3.63(\mathrm{dd}, 10.7,5.8)\end{array}$ & $65.9(\mathrm{t})$ & $\begin{array}{l}3.60(\mathrm{~m}) \\
3.65(\mathrm{~m})\end{array}$ & $65.9(\mathrm{t})$ \\
\hline
\end{tabular}

Multiplicities and coupling constants (in $\mathrm{Hz}$ ) in parentheses.

supported by the molecular formula and the HMBC NMR data of 3 (Fig. 2). Accordingly, the structure of trichurusin $\mathrm{C}$ was deduced to be $\mathbf{3}$, as shown in Fig. 2 .

Trichurusin D (TT-4) (4), obtained as an optically active white amorphous powder $\left([\alpha]_{D}^{25}-78.1^{\circ}\right)$, has $\mathrm{C}_{25} \mathrm{H}_{38} \mathrm{O}_{7}$ as the molecular formula. Comparison of the ${ }^{1} \mathrm{H}$ - and ${ }^{13} \mathrm{C}-\mathrm{NMR}$ spectral data of 4 (Table 2) with those of 1 showed that instead of the signals of $\mathrm{CH}_{3}-16\left[\delta_{\mathrm{H}} 1.53(3 \mathrm{H}, \mathrm{s}), \delta_{\mathrm{C}} 15.5(\mathrm{q})\right]$ and $\mathrm{CH}-12\left[\delta_{\mathrm{H}} 5.12(1 \mathrm{H}, \mathrm{q}), \delta_{\mathrm{C}} 120.0(\mathrm{~d})\right]$, those of $\underline{\mathrm{CH}}_{2}=\mathrm{C}<\left[\delta_{\mathrm{H}} 4.75,5.07\right.$ (each $\left.\left.1 \mathrm{H}, \mathrm{s}\right), \delta_{\mathrm{C}} 109.5(\mathrm{t})\right]$ and $-\mathrm{CH}(-\mathrm{O})-\left[\delta_{\mathrm{H}} 4.12(\mathrm{q}), \delta_{\mathrm{C}} 70.6(\mathrm{~d})\right]$ newly appeared, suggesting that the group $\mathrm{CH}_{3}-\mathrm{CH}=\mathrm{C}\left(\mathrm{CH}_{3}\right)$ - in segment $\mathrm{A}$ of 1 
Table 3. ${ }^{1} \mathrm{H}$ - and ${ }^{13} \mathrm{C}-\mathrm{NMR}$ Data for Trichurusins F, G, $\mathrm{H}$, and $\mathrm{I}$ in $\mathrm{CDCl}_{3}, \delta(\mathrm{ppm})$ from TMS as an Internal Standard

\begin{tabular}{|c|c|c|c|c|c|c|c|c|}
\hline \multirow{2}{*}{ Position } & \multicolumn{2}{|c|}{ Trichurusin F (6) } & \multicolumn{2}{|c|}{ Trichurusin G (7) } & \multicolumn{2}{|c|}{ Trichurusin H (8) } & \multicolumn{2}{|c|}{ Trichurusin I (9) } \\
\hline & $\delta_{\mathrm{H}}$ & $\delta_{\mathrm{C}}$ & $\delta_{\mathrm{H}}$ & $\delta_{\mathrm{C}}$ & $\delta_{\mathrm{H}}$ & $\delta_{\mathrm{C}}$ & $\delta_{\mathrm{H}}$ & $\delta_{\mathrm{C}}$ \\
\hline 2 & & $163.0(\mathrm{~s})$ & & $163.5(\mathrm{~s})$ & & $163.2(\mathrm{~s})$ & & $163.2(\mathrm{~s})$ \\
\hline 3 & $6.23(\mathrm{~d}, 9.3)$ & $125.0(\mathrm{~d})$ & $6.25(\mathrm{~d}, 9.3)$ & $125.0(\mathrm{~d})$ & $6.22(\mathrm{~d}, 9.6)$ & $125.1(\mathrm{~d})$ & $6.22(\mathrm{~d}, 9.6)$ & $125.1(\mathrm{~d})$ \\
\hline 4 & $7.02(\mathrm{~m})$ & $140.3(d)$ & $6.99(\mathrm{~m})$ & $140.3(d)$ & $7.03(\mathrm{dd}, 9.6,6.0)$ & $140.5(\mathrm{~d})$ & $7.02(\mathrm{dd}, 9.6,6.0)$ & $140.4(d)$ \\
\hline 5 & $5.41(\mathrm{brd}, 6.0)$ & 61.5 (d) & $5.48(\mathrm{brd}, 5.5)$ & $61.5(\mathrm{~d})$ & $5.37(\mathrm{dd}, 6.0,2.7)$ & 61.7 (d) & $5.38(\mathrm{dd}, 6.0,2.5)$ & $61.3(d)$ \\
\hline 6 & $4.16(\mathrm{brd}, 6.9)$ & 83.0 (d) & $4.17(\mathrm{brd}, 9.1)$ & 83.0 (d) & $4.15(\mathrm{dd}, 8.7,2.7)$ & 83.0 (d) & $4.11(\mathrm{dd}, 9.3,2.5)$ & $83.3(\mathrm{~d})$ \\
\hline 7 & $2.17(\mathrm{~m})$ & 31.7 (d) & $2.17(\mathrm{~m})$ & 31.7 (d) & $2.09(\mathrm{~m})$ & 31.6 (d) & $2.14(\mathrm{~m})$ & $31.3(\mathrm{~d})$ \\
\hline 8 & $1.11(\mathrm{~m}), 1.26(\mathrm{~m})$ & $39.8(\mathrm{t})$ & $1.11(\mathrm{~m}), 1.26(\mathrm{~m})$ & $39.8(\mathrm{t})$ & $1.03(\mathrm{~m}), 1.25(\mathrm{~m})$ & $40.4(t)$ & $1.06(\mathrm{~m}), 1.20(\mathrm{~m})$ & $40.2(t)$ \\
\hline 9 & $1.85(\mathrm{~m})$ & 28.4 (d) & $1.85(\mathrm{~m})$ & $28.4(\mathrm{~d})$ & $1.77(\mathrm{~m})$ & 27.4 (d) & $1.71(\mathrm{~m})$ & $27.4(d)$ \\
\hline 10 & $\begin{array}{l}1.66(\mathrm{~m}) \\
2.33(\mathrm{dd}, 13.1,4.3)\end{array}$ & $47.4(t)$ & $\begin{array}{l}1.70(\mathrm{~m}) \\
2.36(\mathrm{brd}, 9.9)\end{array}$ & $38.4(t)$ & $\begin{array}{l}1.00(\mathrm{brd}, 12.0) \\
1.66(\mathrm{dd}, 12.0,4.4)\end{array}$ & $44.3(t)$ & $\begin{array}{l}0.54(\mathrm{t}, 12.7) \\
1.97(\mathrm{dd}, 12.7,3.6)\end{array}$ & $45.0(t)$ \\
\hline 11 & & $163.0(\mathrm{~s})$ & & $164.0(\mathrm{~s})$ & & $60.1(\mathrm{~s})$ & & $60.1(\mathrm{~s})$ \\
\hline 12 & $5.78(\mathrm{~d}, 8.0)$ & 129.0 (d) & $5.93(\mathrm{~d}, 8.4)$ & 130.5 (d) & $2.69(\mathrm{q}, 5.5)$ & 58.2 (d) & $2.76(\mathrm{q}, 5.6)$ & $60.5(d)$ \\
\hline 13 & $9.94(\mathrm{~d}, 8.0)$ & $191.2(d)$ & $9.75(\mathrm{~d}, 8.4)$ & $190.8(d)$ & $1.26(3 \mathrm{H}, \mathrm{d}, 5.5)$ & $14.0(\mathrm{q})$ & $1.25(3 \mathrm{H}, \mathrm{d}, 5.6)$ & $14.0(q)$ \\
\hline 14 & $1.18(3 \mathrm{H}, \mathrm{d}, 6.9)$ & $16.0(q)$ & $1.19(3 \mathrm{H}, \mathrm{d}, 6.9)$ & $16.0(q)$ & $1.14(3 \mathrm{H}, \mathrm{d}, 6.6)$ & $16.0(\mathrm{q})$ & $1.15(3 \mathrm{H}, \mathrm{d}, 6.6)$ & $15.7(q)$ \\
\hline 15 & $0.86(3 \mathrm{H}, \mathrm{d}, 6.6)$ & $20.6(q)$ & $0.87(3 \mathrm{H}, \mathrm{d}, 6.6)$ & $20.6(q)$ & $0.97(3 \mathrm{H}, \mathrm{d}, 6.6)$ & $21.5(\mathrm{q})$ & $0.87(3 \mathrm{H}, \mathrm{d}, 6.6)$ & $21.0(\mathrm{q})$ \\
\hline 16 & $2.12(3 \mathrm{H}, \mathrm{s})$ & $17.3(\mathrm{q})$ & $1.93(3 \mathrm{H}, \mathrm{s})$ & $24.7(q)$ & $1.22(3 \mathrm{H}, \mathrm{s})$ & $16.6(q)$ & $1.19(3 \mathrm{H}, \mathrm{s})$ & $16.2(q)$ \\
\hline $1^{\prime}$ & & $166.2(\mathrm{~s})$ & & $166.2(\mathrm{~s})$ & & $166.1(\mathrm{~s})$ & & $166.2(\mathrm{~s})$ \\
\hline $2^{\prime}$ & $5.81(\mathrm{~d}, 15.4)$ & 114.8 (d) & $5.81(\mathrm{~d}, 15.6)$ & 114.8 (d) & $5.80(\mathrm{~d}, 15.3)$ & 115.0 (d) & $5.79(\mathrm{~d}, 15.7)$ & 114.8 (d) \\
\hline $3^{\prime}$ & $7.38(\mathrm{~d}, 15.4)$ & $151.5(\mathrm{~d})$ & $7.41(\mathrm{~d}, 15.6)$ & $151.7(\mathrm{~d})$ & $7.34(\mathrm{~d}, 15.3)$ & $151.2(\mathrm{~d})$ & $7.32(\mathrm{~d}, 15.7)$ & $151.5(d)$ \\
\hline $4^{\prime}$ & & $134.6(\mathrm{~s})$ & & $134.6(\mathrm{~s})$ & & $134.7(\mathrm{~s})$ & & $134.9(\mathrm{~s})$ \\
\hline $5^{\prime}$ & $5.84(\mathrm{~d}, 11.5)$ & $144.3(d)$ & $5.84(\mathrm{~d}, 11.5)$ & $144.3(d)$ & $5.80(\mathrm{~d}, 10.7)$ & $143.7(\mathrm{~d})$ & $5.79(\mathrm{~d}, 12.1)$ & $144.0(d)$ \\
\hline $6^{\prime}$ & $2.89(\mathrm{~m})$ & $39.2(d)$ & $2.89(\mathrm{~m})$ & $39.2(\mathrm{~d})$ & $2.89(\mathrm{~m})$ & $39.3(d)$ & $2.89(\mathrm{~m})$ & $39.4(d)$ \\
\hline $7^{\prime}$ & $1.61(\mathrm{~m}), 1.81(\mathrm{~m})$ & $34.7(\mathrm{t})$ & $1.61(\mathrm{~m}), 1.81(\mathrm{~m})$ & $34.7(t)$ & $1.61(\mathrm{~m}), 1.83(\mathrm{~m})$ & $34.8(t)$ & $1.60(\mathrm{~m}), 1.80(\mathrm{~m})$ & $34.6(\mathrm{t})$ \\
\hline $8^{\prime}$ & $3.59(\mathrm{~m}), 3.71(\mathrm{~m})$ & $60.7(t)$ & $3.59(\mathrm{~m}), 3.71(\mathrm{~m})$ & $60.7(\mathrm{t})$ & $3.61(\mathrm{~m}), 3.73(\mathrm{~m})$ & $60.7(t)$ & $3.61(\mathrm{~m}), 3.70(\mathrm{~m})$ & $60.6(\mathrm{t})$ \\
\hline $9^{\prime}$ & $1.84(3 \mathrm{H}, \mathrm{s})$ & $12.7(\mathrm{q})$ & $1.81(3 \mathrm{H}, \mathrm{s})$ & $12.7(q)$ & $1.85(3 \mathrm{H}, \mathrm{s})$ & $12.7(\mathrm{q})$ & $1.85(3 \mathrm{H}, \mathrm{s})$ & $12.8(\mathrm{q})$ \\
\hline $10^{\prime}$ & $\begin{array}{l}3.61(\mathrm{~m}) \\
3.64(\mathrm{~m})\end{array}$ & $65.9(\mathrm{t})$ & $\begin{array}{l}3.61(\mathrm{~m}), \\
3.64(\mathrm{~m})\end{array}$ & $65.9(\mathrm{t})$ & $\begin{array}{l}3.60(\mathrm{dd}, 10.4,6.2), \\
3.63(\mathrm{dd}, 10.4,6.9)\end{array}$ & $65.9(t)$ & $\begin{array}{l}3.57(\mathrm{dd}, 11.0,6.7), \\
3.63(\mathrm{dd}, 11.0,5.8)\end{array}$ & $66.1(\mathrm{t})$ \\
\hline
\end{tabular}

Multiplicities and coupling constants (in $\mathrm{Hz}$ ) in parentheses.

was replaced with $\mathrm{CH}_{3}-\mathrm{CH}(\mathrm{OH})-\mathrm{C}\left(=\mathrm{CH}_{2}\right)$ - in that of 4 . This was also supported by the molecular formula and the HMBC NMR data of 4 (Fig. 2). Accordingly, the structure of trichurusin $\mathrm{D}$ was deduced to be $\mathbf{4}$, as shown in Fig. 2.

Trichurusin E (TT-5) (5) was obtained as a white amorphous powder. Comparison of the ${ }^{13} \mathrm{C}$-NMR spectral data of 5 (Table 2) with those of $\mathbf{3}$ showed that all of the signals of $\mathbf{5}$ were quite similar to those of $\mathbf{3}$ except that the signals of C$10,-11,-12,-13$, and -16 were shifted to $\delta_{\mathrm{C}} 44.1(-4.0)$, $84.8(+11.1), 141.8(-3.4), 115.3(+3.6)$, and $21.6(-7.6)$, respectively, suggesting that $\mathbf{5}$ might be the epimer of $\mathbf{3}$ at position 11, as shown in Fig. 2. This was also supported by the fact that the signals of $\mathrm{H}_{2}-10, \mathrm{H}_{2}-13$, and $\mathrm{H}_{3}-16$ were only shifted in comparison to the ${ }^{1} \mathrm{H}-\mathrm{NMR}$ spectral data of $\mathbf{5}$ (Table 2) with those of $\mathbf{3}$. Though other physicochemical properties of 5 remained unclear because this compound was gradually decomposed while standing at room temperature, we proposed the tentative structure as shown in Fig. 2 to $\mathbf{5}$.

A mixture of trichurusins F (TT-6) (6) and G (TT-7) (7) was obtained as a uniformly white amorphous powder, which was estimated to be a mixture of $\mathbf{6}$ and its stereoisomer (7) in the ratio of $c a .3$ to 2 by the ${ }^{1} \mathrm{H}$ - and ${ }^{13} \mathrm{C}$-NMR spectral data (Table 3). The mixture of 6 and $7(6 / 7)$ gave $\mathrm{C}_{25} \mathrm{H}_{36} \mathrm{O}_{7}$ as their common molecular formulae. In the ${ }^{1} \mathrm{H}$ - and ${ }^{13} \mathrm{C}-\mathrm{NMR}$ spectra, each signal due to $\mathbf{6}$ was independently observed from each corresponding one due to 7. Comparison of the ${ }^{1} \mathrm{H}$ - and ${ }^{13} \mathrm{C}$-NMR spectral data of both $\mathbf{6}$ and 7 with those of 1 showed that instead of the signal of $\mathrm{CH}_{3}-13$, those of CHO13 of $6\left[\delta_{\mathrm{H}} 9.94(\mathrm{~d}), \delta_{\mathrm{C}} 191.2(\mathrm{~d})\right]$ and $7\left[\delta_{\mathrm{H}} 9.75(\mathrm{~d}), \delta_{\mathrm{C}}\right.$ 190.8 (d)] newly appeared, respectively, suggesting that the group $\mathrm{CH}_{3}-\mathrm{CH}=\mathrm{C}\left(\mathrm{CH}_{3}\right)$ - in segment $\mathrm{A}$ of $\mathbf{1}$ was replaced with $\mathrm{CHO}-\mathrm{CH}=\mathrm{C}\left(\mathrm{CH}_{3}\right)-$ in that of both 6 and 7. This was also supported by the common molecular formulae and the HMBC NMR data (Fig. 3). In the differential nuclear Overhauser effect (DifNOE) experiment, $2.3 \%$ of NOE was observed between $\mathrm{CHO}-13\left(\delta_{\mathrm{H}} 9.94\right)$ and $\mathrm{CH}_{3}-16\left[\delta_{\mathrm{H}} 2.12(3 \mathrm{H}\right.$, s)] of 6 , meanwhile, $4.8 \%$ of NOE was observed between CHO-13 $\left(\delta_{\mathrm{H}} 9.75\right)$ and one $\mathrm{H}$ of $\mathrm{CH}_{2}-10\left[\delta_{\mathrm{H}} 2.36(1 \mathrm{H}, \mathrm{brd})\right]$ of 7, indicating that the configuration at position 11 might be $E$ in 6, but $Z$ in 7. Accordingly, the structures of trichurusins $\mathrm{F}$ and $\mathrm{G}$ were deduced to be $\mathbf{6}$ and 7 , respectively, as shown in Fig. 3.

Trichurusin H (TT-8) (8), obtained as an optically active white amorphous powder $\left([\alpha]_{D}^{25}-124.9^{\circ}\right)$, has $\mathrm{C}_{25} \mathrm{H}_{38} \mathrm{O}_{7}$ as its molecular formula. Comparison of the ${ }^{1} \mathrm{H}-$ and ${ }^{13} \mathrm{C}-\mathrm{NMR}$ spectral data of $\mathbf{8}$ (Table 3) with those of $\mathbf{1}$ revealed that large shifts were observed on the signals of $\mathrm{C}-11\left[\delta_{\mathrm{C}} 60.1\right.$ $(-74.0)]$ and $\mathrm{CH}-12\left[\delta_{\mathrm{H}} 2.69(-2.43), \delta_{\mathrm{C}} 58.2(-61.8)\right]$, and on the signals of $\mathrm{CH}_{2}-10\left[\delta_{\mathrm{H}} 1.00(\mathrm{brd}), 1.66(\mathrm{dd})\right]$, $\mathrm{C}_{3}-13\left[\delta_{\mathrm{H}} 1.26(3 \mathrm{H}, \mathrm{d})\right]$, and $\mathrm{C}_{3}-16\left[\delta_{\mathrm{H}} 1.22(3 \mathrm{H}, \mathrm{s})\right]$, suggesting that instead of the $\mathrm{C}=\mathrm{C}$ bond at position 11(12), an epoxy ring might be present between $\mathrm{C}-11$ and $\mathrm{C}-12$ of 8 . This was also supported by the molecular formula and the HMBC NMR data (Fig. 3). Accordingly, the structure of trichurusin $\mathrm{H}$ was deduced to be $\mathbf{8}$, as shown in Fig. 3 .

Trichurusin I (TT-9) (9), obtained as an optically active white amorphous powder $\left([\alpha]_{\mathrm{D}}^{25}-167.3^{\circ}\right)$, gave $\mathrm{C}_{25} \mathrm{H}_{38} \mathrm{O}_{7}$ as the molecular formula, indicating that $\mathbf{9}$ was an isomer of $\mathbf{8}$. Comparison of the ${ }^{1} \mathrm{H}-$ and ${ }^{13} \mathrm{C}-\mathrm{NMR}$ spectral data of 9 (Table 3) with those of $\mathbf{8}$ showed that all of the signals of $\mathbf{9}$ were quite similar to those of $\mathbf{8}$ except for the presence of considerably large shifts in the signals of $\mathrm{CH}_{2}-10\left[\delta_{\mathrm{H}} 0.54\right.$ 

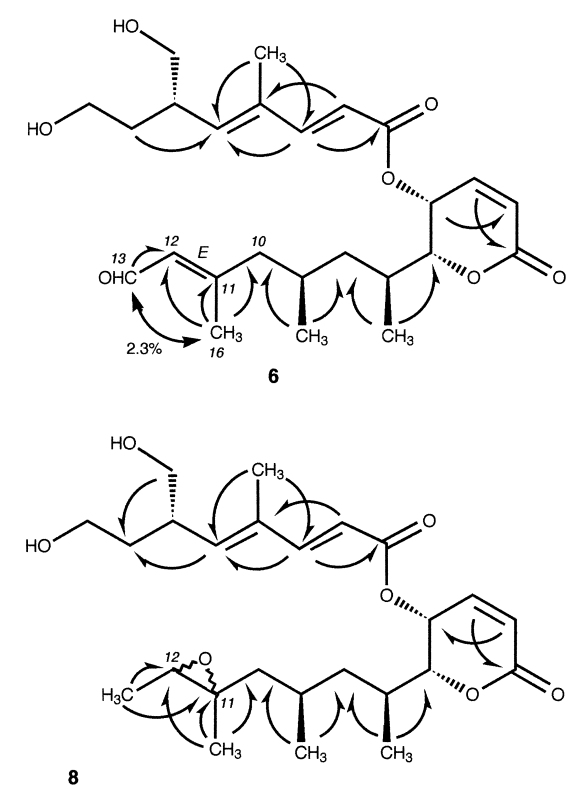

9 (may be a diastereomer of 8 at positions 11 and 12)
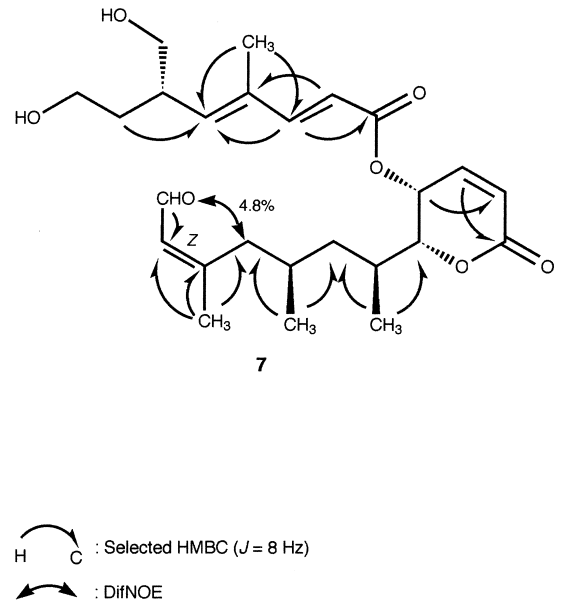

Fig. 3

$\left.(-0.46), 1.97(+0.31), \delta_{\mathrm{C}} 45.0(+0.7)\right]$ and $\mathrm{CH}-12\left[\delta_{\mathrm{H}} 2.76\right.$ $(+0.07), \delta_{\mathrm{C}} 60.5(+2.3)$ ], suggesting that 9 might be a diastereomer of $\mathbf{8}$ concerning the configuration of the epoxy ring between $\mathrm{C}-11$ and $\mathrm{C}-12$. This was also supported by the HMBC NMR data (Fig. 3). Accordingly, the structure of trichurusin I was deduced to be $\mathbf{9}$, as shown in Fig. 3.

Trichurusin J (TT-10) (10), obtained as an optically active colorless amorphous powder $\left([\alpha]_{\mathrm{D}}^{24}-135.1^{\circ}\right)$, gave $\mathrm{C}_{27} \mathrm{H}_{40} \mathrm{O}_{7}$ as the molecular formula. Comparison of the ${ }^{1} \mathrm{H}$ - and ${ }^{13} \mathrm{C}-$ NMR spectral data of $\mathbf{1 0}$ (Table 1) with those of $\mathbf{1}$ showed that the signal of $\mathrm{CH}_{3} \mathrm{CO}$ newly appeared at $\delta_{\mathrm{H}} 2.02(3 \mathrm{H}, \mathrm{s})$, $\delta_{\mathrm{C}} 21.0(\mathrm{q})$, and $171.0(\mathrm{~s})$, and the signals of $\mathrm{C}-8^{\prime}$ and $-7^{\prime}$ were shifted to $\delta_{\mathrm{C}} 62.3(+1.9)$ and $30.4(-4.4)$, respectively, indicating that, considering the acetylation shift rule, ${ }^{4,5)} \mathbf{1 0}$ might be an $8^{\prime}-O$-acetyl ester of $\mathbf{1}$. This was also supported by the molecular formula. Accordingly, the structure of trichurusin $\mathrm{J}$ was deduced to be 10, as shown in Fig. 1.

Trichurusin K (TT-11) (11), obtained as an optically active colorless amorphous powder $\left([\alpha]_{D}^{25}-122.3^{\circ}\right)$, gave $\mathrm{C}_{27} \mathrm{H}_{40} \mathrm{O}_{7}$ as the molecular formula. Comparison of the ${ }^{1} \mathrm{H}-$ and ${ }^{13} \mathrm{C}-$ NMR spectral data of $\mathbf{1 1}$ (Table 1) with those of $\mathbf{1}$ showed that the signal of $\mathrm{CH}_{3} \mathrm{CO}$ newly appeared at $\delta_{\mathrm{H}} 2.02(3 \mathrm{H}, \mathrm{s})$, $\delta_{\mathrm{C}} 20.9(\mathrm{q})$, and $171.0(\mathrm{~s})$, and the signals of $\mathrm{C}-10^{\prime}$ and $-6^{\prime}$ were shifted to $\delta_{\mathrm{C}} 66.9(+1.3)$ and $35.1(-4.1)$, respectively, indicating that, $\mathbf{1 1}$ might be a $10^{\prime}-O$-acetyl ester of $\mathbf{1}$. This was also supported by the molecular formula. Accordingly, the structure of trichurusin $\mathrm{K}$ was deduced to be 11, as shown in Fig. 1.

The fact that the specific rotations and the CD curves of trichurusins C (3), D (4), the mixture of trichurusins F and G (6/7), H (8), I (9), J (10), and K (11) (see Experimental) were similar to those of $\mathbf{1}$ suggested that the absolute configurations at positions $5,6,7,9$, and $6^{\prime}$ in $\mathbf{3}, \mathbf{4}, \mathbf{6}, \mathbf{7}, \mathbf{8}, \mathbf{9}, \mathbf{1 0}$, and 11 might be the same as those in $1\left(5 R, 6 R, 7 S, 9 R\right.$, and $\left.6^{\prime} S\right)$, as shown in Figs. 1, 2, and 3. Though the optical data of trichurusin E (5) were not obtained from its decomposition, $\mathbf{5}$ was considered to be the epimer of $\mathbf{3}$ at position 11 from the ${ }^{1} \mathrm{H}$ - and ${ }^{13} \mathrm{C}-\mathrm{NMR}$ spectral data as mentioned above. Mean-
Table 4. Immunosuppressive Effects of TT-1 (Rasfonin), Trichurusins BD, H-K, TT-1 (Rasfonin) Diacetate, and Azathioprine, Cyclosporin A, and Tacrolimus (FK506) on the Con A-Induced and LPS-Induced Proliferations of Mouse Splenic Lymphocytes

\begin{tabular}{lll}
\hline \hline \multirow{2}{*}{ Compound } & \multicolumn{2}{c}{$\mathrm{IC}_{50}(\mu \mathrm{g} / \mathrm{ml})$} \\
\cline { 2 - 3 } & Con A-induced & LPS-induced \\
\hline TT-1 (Rasfonin) (1) & 0.7 & 0.5 \\
Trichurusin B (2) & 2.0 & 0.8 \\
Trichurusin C (3) & 1.2 & 0.4 \\
Trichurusin D (4) & 0.9 & 0.4 \\
Trichurusin H (8) & 6.2 & 6.0 \\
Trichurusin I (9) & 4.7 & 4.5 \\
Trichurusin J (10) & $n . t$. & 6.4 \\
Trichurusin K (11) & 6.9 & 6.9 \\
TT-1 (Rasfonin) diacetate (12) & 6.0 & 6.9 \\
Azathioprine & 2.7 & 2.7 \\
Cyclosporin A & 0.04 & 0.07 \\
Tacrolimus (FK506) & $1.5 \times 10^{-5}$ & $1.6 \times 10^{-3}$ \\
& & \\
\hline
\end{tabular}

The $\mathrm{IC}_{50}$ value of each sample was calculated from the correlation curve between the sample concentration (horizontal axis) and the cell proliferation (vertical axis). The curve of each sample was drawn with 7 points, each of which represented the mean of three experiments on each correlation between 7 different concentrations and cell proliferations. n.t.: not tested.

while, the absolute stereochemistry of trichurusin B (2) remained pending, because the specific rotation and the CD curve of $\mathbf{2}$ (see Experimental) were different from those of $\mathbf{1}$.

The immunosuppressive activities $\left(\mathrm{IC}_{50}\right.$ values) of $\mathbf{1}-\mathbf{4}$, 8-11, and diacetate of $\mathbf{1}$ (12) were calculated against Con A-induced ( $\mathrm{T}$ cell) and LPS-induced (B cell) proliferations of mouse splenic lymphocytes, as shown in Table 4. Comparison of the $\mathrm{IC}_{50}$ values of these new compounds with those of some known immunosuppressants in Table 4 showed that 1-4 possessed considerably high immunosuppressive activities, while 8-11 possessed moderate ones. The fact that the immunosuppressive activities of $\mathbf{1 0}, \mathbf{1 1}$ and $\mathbf{1 2}$ were lower than that of 1 suggests that the presence of both free $\mathrm{OH}$ groups at positions $8^{\prime}$ and $10^{\prime}$ might be important for the appearance of the immunosuppressive activity of $\mathbf{1}$. 


\section{Experimental}

The general procedures for chemical experiments and other experimental conditions, including those for the evaluation of suppressive activity $\left(\mathrm{IC}_{50}\right.$ values) of samples against the proliferation of mouse splenic lymphocytes stimulated with Con A and LPS, were the same as those described in our previous reports [this method is based on the formation ratio of MTT-formazan from exogenous 3-(4,5-dimethyl-2-thiazolyl)-2,5-diphenyl- $2 H$-tetrazolium bromide (MTT) in lymphocytes]. ${ }^{2}$ Chemical shifts are expressed in $\delta(\mathrm{ppm})$ values from tetramethylsilane (TMS) as an internal standard.

Isolation of Rasfonin (1) and Trichurusins B-D (2-4) from T. terrophilus IFM4606 T. terrophilus IFM4606 $6^{3)}$ was cultivated on sterilized moistened-rice in Roux flasks $(200 \mathrm{~g} /$ flask $\times 85)$ at $25^{\circ} \mathrm{C}$ for $20 \mathrm{~d}$. The whitish gray moldy-rice was extracted with EtOAc (201) with shaking at room temperature for $6 \mathrm{~h}$ twice to give an EtOAc solution ( $c a .401)$, which gave, after evaporation in vacuo, an EtOAc extract (32.44 g). A concentrated solution of the EtOAc extract in $\mathrm{MeOH}(30 \mathrm{ml})$ was suspended in $\mathrm{H}_{2} \mathrm{O}$ $(600 \mathrm{ml})$. The suspension was partitioned with $n$-hexane $(600 \mathrm{ml})$ twice into an $n$-hexane layer (after evaporation in vacuo, $23.75 \mathrm{~g}$ ) and an aqueous suspension. The aqueous suspension was further partitioned with EtOAc $(600 \mathrm{ml})$ twice into an EtOAc layer $(5.63 \mathrm{~g})$ and an aqueous layer $(0.75 \mathrm{~g})$. The $\mathrm{IC}_{50}$ values of the $n$-hexane, EtOAc, and aqueous layers against the Con A-induced proliferation of mouse splenic lymphocytes were 9.7, 2.5, and $>50 \mu \mathrm{g} / \mathrm{ml}$, respectively. The EtOAc layer was subjected to chromatography on a silica gel (Wakogel C-200, Wako) column with $n$-hexane-EtOAc $(15: 1, \mathrm{v} / \mathrm{v}),(7.5: 1),(5: 1),(2: 1),(1: 3), \mathrm{EtOAc}$, and $\mathrm{MeOH}$ to give seven fractions $1 \mathrm{a}-\mathrm{g}(73,99,758,714,431,479,987 \mathrm{mg})$, whose $\mathrm{IC}_{50}$ values against the Con A-induced proliferation were 39.3, 19.3, 22.1, 1.6, 1.8, 1.2, and $18.7 \mu \mathrm{g} / \mathrm{ml}$, respectively. Fraction $1 \mathrm{f}$ was further chromatographed on a silica gel column with $\mathrm{CHCl}_{3}-\mathrm{MeOH}(50: 1),(37.5: 1),(17.5: 1),(10: 1)$, $(7.5: 1)$, and $(2.5: 1)$ to give six fractions $2 \mathrm{a}-\mathrm{f}(19,230,105,70,22$, $13 \mathrm{mg}$ ), respectively. Fraction $2 \mathrm{~b}$ was separated by HPLC on an octadecyl silica gel (ODS) column (Pegasil ODS, Senshu) with $\mathrm{MeOH}-\mathrm{H}_{2} \mathrm{O}(3: 1)$ at a flow rate of $8.0 \mathrm{ml} / \mathrm{min}$ to afford seven fractions $3 \mathrm{a}-\mathrm{g}(25.0,5.9,83.6,13.3$, $4.1,2.5,19.8 \mathrm{mg}$ ), respectively. Fractions $3 \mathrm{c}$ and $\mathrm{f}$ directly afforded 1 $(83.6 \mathrm{mg})$ and $2(2.5 \mathrm{mg})$, respectively. Fraction $3 \mathrm{a}$ was further separated by HPLC on a silica gel column (Aquasil SS-652, Senshu) with $\mathrm{CHCl}_{3}-\mathrm{MeOH}$ $(30: 1)$ at a flow rate of $2.0 \mathrm{ml} / \mathrm{min}$ to afford $3(0.9 \mathrm{mg})$ and $4(0.7 \mathrm{mg})$. Fraction $2 \mathrm{c}$ was treated with $\mathrm{MeOH}$ to give a $\mathrm{MeOH}$-soluble portion $(72 \mathrm{mg})$, which was separated by HPLC on an ODS column with $\mathrm{CH}_{3} \mathrm{CN}-\mathrm{H}_{2} \mathrm{O}(3: 7)$ at a flow rate of $8.0 \mathrm{ml} / \mathrm{min}$ to give six fractions $4 \mathrm{a}-\mathrm{f}(7.2,1.8,3.2,9.7,1.8$, $24.5 \mathrm{mg}$ ), respectively. Fraction $4 \mathrm{c}$ was further separated by HPLC on a silica gel column with $\mathrm{CHCl}_{3}-\mathrm{MeOH}(20: 1)$ at a flow rate of $2.0 \mathrm{ml} / \mathrm{min}$ to afford $3(1.3 \mathrm{mg})$ and $4(1.1 \mathrm{mg})$. Fraction $4 \mathrm{~d}$ was also separated by HPLC on an ODS column with $\mathrm{CH}_{3} \mathrm{CN}-\mathrm{H}_{2} \mathrm{O}(1: 3)$ to afford $3(1.3 \mathrm{mg})$ and 4 (1.1 mg).

Isolation of Rasfonin (1) and Trichurusins B-K (2-11) from T. terrophilus IFM46251 T. terrophilus IFM $46251^{3)}$ was cultivated on sterilized moistened-rice in Roux flasks $(200 \mathrm{~g} /$ flask $\times 150)$ at $25^{\circ} \mathrm{C}$ for $20 \mathrm{~d}$. The greenish gray moldy-rice was extracted with EtOAc (401) with shaking at room temperature for $6 \mathrm{~h}$ twice to give an EtOAc extract $(159.8 \mathrm{~g}$ ), which was dissolved in $\mathrm{MeOH}(100 \mathrm{ml})$ and then suspended in $\mathrm{H}_{2} \mathrm{O}(1000 \mathrm{ml})$. The suspension was partitioned with $n$-hexane twice $(750,375 \mathrm{ml})$ into an $n$ hexane layer $(70.2 \mathrm{~g})$ and an aqueous suspension. The aqueous suspension was further partitioned with EtOAc twice $(750,375 \mathrm{ml})$ into an EtOAc layer $(34.9 \mathrm{~g})$ and an aqueous layer $(40.1 \mathrm{~g})$. The EtOAc layer was subjected to chromatography on a silica gel (PSQ100B, Fuji Silysia) column with $n$ hexane $n$-hexane-EtOAc $(3: 1),(1: 1),(1: 3)$, EtOAc, and $\mathrm{MeOH}$ to give five fractions $1 \mathrm{a}-\mathrm{e}(19.78,3.00,1.37,4.41,5.03 \mathrm{~g})$, respectively. Fraction $1 \mathrm{~d}$ was further chromatographed on a silica gel column with $\mathrm{CHCl}_{3}-\mathrm{MeOH}$ $(50: 1),(50: 1),(25: 1),(10: 1),(10: 1)$, and $\mathrm{MeOH}$ to give six fractions $2 \mathrm{a}-\mathrm{f}(176,2593,403,934,83,211 \mathrm{mg})$, respectively. Fraction 2b was separated by chromatography on an ODS (Chromatorex, Fuji Silysia) column with $\mathrm{CH}_{3} \mathrm{CN}-\mathrm{H}_{2} \mathrm{O}(2: 3),(1: 1),(3: 2)$, and $\mathrm{CH}_{3} \mathrm{CN}$ to give four fractions $3 a-d(285,1675,62,230 \mathrm{mg})$, respectively. Fraction $3 \mathrm{~b}$ was separated by HPLC on an ODS column (ODS-5251S, Senshu) with $\mathrm{CH}_{3} \mathrm{CN}-\mathrm{H}_{2} \mathrm{O}(1: 1)$, $(1: 1),(1: 1),(1: 1),(3: 2),(3: 2),(3: 2)$, and $(3: 2)$ at a flow rate of $7.0 \mathrm{ml} / \mathrm{min}$ to afford eight fractions $4 \mathrm{a}-\mathrm{h}(20,10,19,15,11,1328,47$, $36 \mathrm{mg})$, respectively. Fraction $4 \mathrm{f}$ directly afforded 1 (1328 mg). Fractions $4 \mathrm{c}, \mathrm{d}$, and $\mathrm{g}$ were purified by chromatography on silica gel columns with $\mathrm{CHCl}_{3}-\mathrm{MeOH}(30: 1)$ to afford $8(3.0 \mathrm{mg})$, a fraction $4 \mathrm{~d}^{\prime}(8.9 \mathrm{mg})$, and a fraction $4 \mathrm{~g}^{\prime}(13 \mathrm{mg})$, respectively. Fractions $4 \mathrm{~d}^{\prime}$ and $\mathrm{g}^{\prime}$ were further purified by HPLC on ODS columns with $\mathrm{CH}_{3} \mathrm{CN}-\mathrm{H}_{2} \mathrm{O}(1: 1)$ to afford $9(5.8 \mathrm{mg})$ and $2(1.4 \mathrm{mg})$, respectively. Fraction $2 \mathrm{~d}$ was separated by chromatography on an ODS column with $\mathrm{CH}_{3} \mathrm{CN}-\mathrm{H}_{2} \mathrm{O}(2: 3),(2: 3),(2: 3),(2: 3),(1: 1)$, $(1: 1)$, and $\mathrm{CHCl}_{3}$ to give seven fractions $5 \mathrm{a}-\mathrm{g}(69,159,343,39,26,26$, $158 \mathrm{mg}$ ), respectively. Fraction $5 \mathrm{c}$ was further chromatographed by HPLC on an ODS column with $\mathrm{CH}_{3} \mathrm{CN}-\mathrm{H}_{2} \mathrm{O}(2: 3)$ to give six fractions $6 \mathrm{a}-\mathrm{f}(5$, $15,16,23,38,118 \mathrm{mg})$, respectively. Fraction 6 a directly afforded 4 $(5.0 \mathrm{mg})$. Fractions $6 \mathrm{~b}, \mathrm{c}$, and $\mathrm{d}$ were purified by HPLC on ODS columns with $\mathrm{CH}_{3} \mathrm{CN}-\mathrm{H}_{2} \mathrm{O}(2: 3)$ to afford $\mathbf{3}(10 \mathrm{mg})$, both $3(4.6 \mathrm{mg})$ and $4(2.0 \mathrm{mg})$, and both $5(4.0 \mathrm{mg})$ and $6 / 7(1.5 \mathrm{mg})$, respectively. The $n$-hexane layer was subjected to chromatography on a silica gel column with $n$-hexane $\sim n$ hexane-EtOAc $(1: 1),(1: 3),(1: 6), \quad$ EtOAc $\sim$ EtOAc-MeOH $(15: 1)$, $(7.5: 1),(3.5: 1)-(1: 1)$, and $\mathrm{MeOH}$ to give seven fractions $7 \mathrm{a}-\mathrm{g}(4.80$, $6.98,3.51,3.11,2.20,1.86,1.13 \mathrm{~g}$ ), respectively. Fraction $7 \mathrm{c}$ was further chromatographed on an ODS column with $\mathrm{CH}_{3} \mathrm{CN}-\mathrm{H}_{2} \mathrm{O}(2: 3),(2: 3)$, $(2: 3),(1: 1),(3: 2),(3: 2), \mathrm{CH}_{3} \mathrm{CN} \sim \mathrm{CHCl}_{3}$ to give seven fractions $8 \mathrm{a}-\mathrm{g}$ $(171,52,1405,31,58,284,552 \mathrm{mg})$, respectively. Fraction $8 \mathrm{c}$ directly afforded $1(1405 \mathrm{mg})$. Fraction 8e was separated by HPLC on an ODS column (Develosil ODS-SR-5, Nomura) with $\mathrm{CH}_{3} \mathrm{CN}-\mathrm{H}_{2} \mathrm{O}(7: 3)$ at a flow rate of $1.8 \mathrm{ml} / \mathrm{min}$ to give four fractions $9 \mathrm{a}-\mathrm{d}(1.1,3.2,24.9,5.2 \mathrm{mg})$, respectively. Fractions $9 \mathrm{~b}$ and $\mathrm{c}$ were separated by preparative TLC on silica gel plates (Kieselgel 60, Merck) with $\mathrm{CHCl}_{3}-\mathrm{MeOH}(20: 1)$ to afford $10(1.8 \mathrm{mg})$ and $11(1.4 \mathrm{mg})$

Rasfonin (1): White amorphous powder (lit., ${ }^{7)}$ colorless oil), $[\alpha]_{\mathrm{D}}^{24}$ $-223.6^{\circ}(c=6.00, \mathrm{MeOH})$ (lit. $\left.\left.{ }^{7}\right)-170^{\circ}(\mathrm{MeOH})\right)$. HR-FAB-MS $\mathrm{m} / \mathrm{z}$ : 435.2736 (Calcd for $\mathrm{C}_{25} \mathrm{H}_{39} \mathrm{O}_{6}\left[(\mathrm{M}+\mathrm{H})^{+}\right]$: 435.2747) (lit. ${ }^{7)}$ 435.2754). UV $\lambda_{\max }^{\mathrm{MeOH}} \mathrm{nm}(\log \varepsilon): 202$ (4.36), 270 (4.49) (lit. ${ }^{7)} 202$ (4.21), 270 (4.36)). IR $v_{\max }^{\mathrm{KBr}} \mathrm{cm}^{-1}: 3437,2926,1715,1621,1258,1158$ (lit. $\left.{ }^{7}\right) 3450,1730$ ). CD (0.11 mм, MeOH) $\Delta \varepsilon(\mathrm{nm}):-98$ (266), -51 (239), -255 (212).

Trichurusin B (2): Colorless amorphous powder, $[\alpha]_{\mathrm{D}}^{25}+18.4^{\circ}(c=0.71$, $\mathrm{MeOH}$ ). HR-FAB-MS m/z: 437.2878 (Calcd for $\mathrm{C}_{25} \mathrm{H}_{41} \mathrm{O}_{6}\left[(\mathrm{M}+\mathrm{H})^{+}\right]$: 437.2904). UV $\lambda_{\max }^{\mathrm{MeOH}} \mathrm{nm}(\log \varepsilon): 203$ (4.19), 268 (4.35). CD (0.062 mM, $\mathrm{MeOH}) \Delta \varepsilon(\mathrm{nm}):+0.34(285),-0.40(253),+4.9(211)$.

Trichurusin $\mathrm{C}(3)$ : Colorless amorphous powder, $[\alpha]_{\mathrm{D}}^{25}-166.6^{\circ}(c=1.08$, $\mathrm{MeOH})$. HR-FAB-MS $m / z$ : 473.2541 (Calcd for $\mathrm{C}_{25} \mathrm{H}_{38} \mathrm{O}_{7} \mathrm{Na}\left[(\mathrm{M}+\mathrm{Na})^{+}\right]$: 473.2516). UV $\lambda_{\max }^{\mathrm{MeOH}} \mathrm{nm}(\log \varepsilon): 206$ (4.32), 271 (4.37). CD (0.094 mM, $\mathrm{MeOH}) \Delta \varepsilon(\mathrm{nm}):-64(269),-40(238),-214(212)$.

Trichurusin D (4): White amorphous powder, $[\alpha]_{\mathrm{D}}^{25}-78.1^{\circ}(c=1.44$, $\mathrm{MeOH}$ ). HR-FAB-MS $m / z: 473.2539$ (Calcd for $\mathrm{C}_{25} \mathrm{H}_{38} \mathrm{O}_{7} \mathrm{Na}\left[(\mathrm{M}+\mathrm{Na})^{+}\right]$: 473.2515). UV $\lambda_{\max }^{\mathrm{MeOH}} \mathrm{nm}(\log \varepsilon): 203$ (4.18), 270 (4.29). CD (0.12 mM, $\mathrm{MeOH}) \Delta \varepsilon(\mathrm{nm}):-32(264),-17(241),-100(212)$.

Trichurusin E (5): White amorphous powder.

Mixture of Trichurusins F and G (6/7) (in the Ratio of $c a .3$ to 2): Uniformly white amorphous powder, $[\alpha]_{\mathrm{D}}^{25}-73.9^{\circ}(c=0.45, \mathrm{MeOH})$. HR-FABMS $m / z: 472.2421$ (Calcd for $\mathrm{C}_{25} \mathrm{H}_{37} \mathrm{O}_{7} \mathrm{Na}\left[(\mathrm{M}+\mathrm{H}+\mathrm{Na})^{+}\right]$: 472.2437$)$. UV $\lambda_{\max }^{\mathrm{MeOH}} \mathrm{nm}(\log \varepsilon): 206$ (4.17), 241 (sh, 4.02), 269 (4.10). CD $(0.17 \mathrm{~mm}$, $\mathrm{MeOH}) \Delta \varepsilon(\mathrm{nm}):-56(266),-2.4(241),-133(211)$.

Trichurusin $\mathrm{H}(\mathbf{8})$ : White amorphous powder, $[\alpha]_{\mathrm{D}}^{25}-124.9^{\circ}(c=0.80$, $\mathrm{MeOH}$ ). HR-FAB-MS $\mathrm{m} / \mathrm{z}$ : 451.2677 (Calcd for $\mathrm{C}_{25} \mathrm{H}_{39} \mathrm{O}_{7}\left[(\mathrm{M}+\mathrm{H})^{+}\right]$: 451.2696). UV $\lambda_{\max }^{\mathrm{MeOH}} \mathrm{nm}(\log \varepsilon): 205$ (4.38), 270 (4.31). CD (0.092 mM, $\mathrm{MeOH}) \Delta \varepsilon(\mathrm{nm}):-66(271),-39(241),-259(214)$.

Trichurusin I (9): White amorphous powder, $[\alpha]_{\mathrm{D}}^{25}-167.3^{\circ}(c=2.9$, $\mathrm{MeOH}$ ). HR-FAB-MS m/z: 451.2691 (Calcd for $\mathrm{C}_{25} \mathrm{H}_{39} \mathrm{O}_{7}\left[(\mathrm{M}+\mathrm{H})^{+}\right]$: 451.2695). UV $\lambda_{\max }^{\mathrm{MeOH}} \mathrm{nm}(\log \varepsilon): 204$ (4.33), 272 (4.37). CD (0.13 mм, $\mathrm{MeOH}) \Delta \varepsilon(\mathrm{nm}):-69(273),-40(239),-232(212)$.

Trichurusin J (10): Colorless amorphous powder, $[\alpha]_{\mathrm{D}}^{24}-135.1^{\circ}(c=0.17$, $\mathrm{MeOH}$ ). HR-FAB-MS m/z: 477.2839 (Calcd for $\mathrm{C}_{27} \mathrm{H}_{41} \mathrm{O}_{7}\left[(\mathrm{M}+\mathrm{H})^{+}\right]$: 477.2852). $\mathrm{CD}$ (0.11 mm, MeOH) $\Delta \varepsilon(\mathrm{nm}):-9.2(265),-5.4(240),-31.1$ (212).

Trichurusin K (11): Colorless amorphous powder, $[\alpha]_{\mathrm{D}}^{25}-122.3^{\circ}$ $\left(c=0.23, \mathrm{MeOH}\right.$ ). HR-FAB-MS $m / z: 477.2822$ (Calcd for $\mathrm{C}_{27} \mathrm{H}_{41} \mathrm{O}_{7}$ $\left.\left[(\mathrm{M}+\mathrm{H})^{+}\right]: 477.2852\right) \cdot \mathrm{CD}(0.090 \mathrm{~mm}, \mathrm{MeOH}) \Delta \varepsilon(\mathrm{nm}):+0.24(325),-8.2$ (264), -4.3 (240), -21.0 (212).

Acetylation of Rasfonin (1) A solution of $\mathbf{1}(10.6 \mathrm{mg})$ in pyridine $(1.0 \mathrm{ml})$ and acetic anhydride $(0.5 \mathrm{ml})$ was allowed to stand at room temperature for $2.5 \mathrm{~h}$. A small volume of ice-water was added to the reaction mixture, which was then extracted with EtOAc. The EtOAc solution was treated as usual to afford $12(10.9 \mathrm{mg})$.

Rasfonin Diacetate (12): Colorless amorphous powder, $[\alpha]_{\mathrm{D}}^{23}-95.7^{\circ}$ $(c=0.72, \mathrm{MeOH}), \mathrm{CD}(0.12 \mathrm{~mm}, \mathrm{MeOH}) \Delta \varepsilon(\mathrm{nm}):+0.089$ (319), -1.65 (264), $-1.16(248),-19.8(214)$.

Acknowledgements We are grateful to Miss R. Hara of Analysis Center, Chiba University for the HR-FAB-MS measurements. We are also grateful to Mr. T. Asai of our laboratory for his help with the bioassay experiment. 


\section{References and Notes}

1) Present address: Faculty of Pharmaceutical Sciences, Teikyo Heisei University; 2289-23 Uruido, Ichihara, Chiba 290-0193, Japan. Email: fujimoto@thu.ac.jp

2) Fujimoto H., Nakamura E., Okuyama E., Ishibashi M., Chem. Pharm. Bull., 52, 1005-1008 (2004), and the references cited therein.

3) This strain was deposited earlier at Research Institute for Chemobiodynamics, Chiba University (present name: Research Center for Pathogenic Fungi and Microbial Toxicoses, Chiba University). A voucher specimen is also on deposit in our laboratory.

4) Ishii H., Seo S., Tori K., Tozyo T., Yoshimura Y., Tetrahedron Lett., 1977, 1227-1230 (1997).
5) Tori K., "Kagaku No Ryoiki Zokan,” Vol. 125, Nankodo, Tokyo, 1980, pp. $221-245$.

6) Fujimoto H., Sone E., Okuyama E., Ishibashi M., Abstracts of Papers 2, 120th Annual Meeting of the Pharmaceutical Society of Japan, Gifu, March 2000, p. 68.

7) Tomikawa T., Shin-ya K., Furihata K., Kinoshita T., Miyajima A., Seto H., Hayakawa Y., J. Antibiot., 53, 848-850 (2000).

8) Akiyama K., Kawamoto S., Fujimoto H., Ishibashi M., Tetrahedron Lett., 44, 8427-8431 (2003).

9) Akiyama K., Yamamoto S., Fujimoto H., Ishibashi M., Tetrahedron, 61, 1827-1833 (2005). 